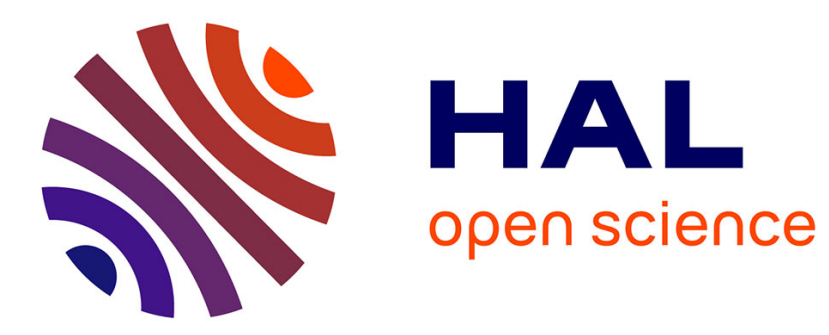

\title{
Analytical solution for a generalized space-time fractional telegraph equation
}

\author{
Ahmad Fino, Hassan Ibrahim
}

\section{To cite this version:}

Ahmad Fino, Hassan Ibrahim. Analytical solution for a generalized space-time fractional telegraph equation. 2011. hal-00613862

\section{HAL Id: hal-00613862 \\ https://hal.science/hal-00613862}

Preprint submitted on 7 Aug 2011

HAL is a multi-disciplinary open access archive for the deposit and dissemination of scientific research documents, whether they are published or not. The documents may come from teaching and research institutions in France or abroad, or from public or private research centers.
L'archive ouverte pluridisciplinaire HAL, est destinée au dépôt et à la diffusion de documents scientifiques de niveau recherche, publiés ou non, émanant des établissements d'enseignement et de recherche français ou étrangers, des laboratoires publics ou privés. 


\title{
Analytical solution for a generalized space-time fractional telegraph equation
}

\author{
Ahmad Z. FINO ${ }^{\mathrm{a}, \mathrm{b}}$, Hassan IBRAHIM ${ }^{\mathrm{c}, \mathrm{d}}$ \\ ${ }^{a}$ LaMA-Liban, Lebanese University, P.O.Box 37 Tripoli, Lebanon \\ ${ }^{b}$ School of Arts and Sciences, Lebanese International University (LIU), Tripoli Campus, Dahr el Ain Road, Tripoly, Lebanon \\ ${ }^{c}$ Lebanese University, Faculty of Sciences-I, Hadath, Lebanon \\ ${ }^{d}$ School of Arts and Sciences, Lebanese International University (LIU), Beirut Campus, Al-Mouseitbeh, P.B. Box 14-6404, Beirut, Lebanon
}

\begin{abstract}
In this paper, we consider a nonhomogeneous space-time fractional telegraph equation defined in a bounded space domain, which is obtained from the standard telegraph equation by replacing the first- or second-order time derivative by the Caputo fractional derivative $D_{t}^{\alpha}, \alpha>0$; and the Laplacian operator by the fractional Laplacian $(-\Delta)^{\beta / 2}$, $\beta \in(0,2]$. We discuss and derive the analytical solutions under nonhomogeneous Dirichlet and Neumann boundary conditions by using the method of separation of variables. The obtained solutions are expressed through multivariate Mittag-Leffler type functions. Special cases of solutions are also discussed.
\end{abstract}

Keywords: Fractional telegraph equation, Fractional Laplacian, Caputo fractional derivative, Multivariate Mittag-Leffler type functions, Method of separating variables

2010 MSC: 35R11, 26A33, 33E12

\section{Introduction and main results}

Fractional differential equations (FDEs) have attracted in the recent years a considerable interest both in mathematics and in applications. For example, fractional derivatives have been used successfully to model frequency dependent damping behavior of many viscoelastic materials, modeling of anomalous diffusive and subdiffusive systems and description of fractional random walk. They are also used in modeling of many chemical processed, mathematical biology and many other problems in engineering (see e.g. $[2,4,7,10,11,19,20]$ ). We offer a simple heuristic discussion to motivate the use of fractional derivatives. Let $X_{1}, \ldots, X_{n}, \ldots$ be independent, identically distributed symmetric random variables with the property that for each $n=1,2, \ldots$ and for suitable positive constants $a_{n}, a_{n} \sum_{j=1}^{n} X_{j}$ has the same distribution as $X_{1}$, and this holds for all $n$. Thus the distribution of $\sum_{i=1}^{n} X_{i}$, suitably scaled, is that of $X_{1}$. This is a kind of self-similarity, independent of the sample size. Necessarily (see [16]) the distribution of $X_{1}$ has Fourier transform $e^{-c \mid \xi^{\beta}}$, for $\xi \in \mathbb{R}$, for certain constants $c>0$ and $\beta \in(0,2]$. These are precisely the symmetric stable laws, including the normal distribution $(\beta=2)$ and the Cauchy distribution $(\beta=1)$. The infinitesimal generator of the corresponding Feller-Markov semigroup is given by a positive multiple of the fractional Laplacian $-\left(-d^{2} / d x^{2}\right)^{\beta / 2}$. The history and a comprehensive treatment of FDEs are provided by Podlubny [25] and a review of some applications of FDEs are given by Mainardi [20].

Roughly speaking, FDEs can be classified into three principal kinds: space-fractional equation, time-fractional equation and space-time fractional equation. One of the interest FDEs is the study of the space-time fractional telegraph equation which is a linear integro partial differential equation obtained from the classical telegraph equation by replacing the time and space derivative terms by fractional derivatives, for example by replacing the first- or second-order time derivative by the Caputo fractional derivative $D_{t}^{\alpha}, \alpha>0$ and the Laplacian operator by the fractional Laplacian $(-\Delta)^{\beta / 2}, \beta \in(0,2]$. This kind of equations has recently been considered by many authors. Cascaval et al. [8] discussed the time-fractional telegraph equations, dealing with well-posedness and presenting a study involving

Email addresses: ahmad.fino01@gmail.com, ahmad.fino@liu.edu.lb (Ahmad Z. FINO), hassan.ibrahim@liu.edu.lb (Hassan IBRAHIM) 
asymptotic by using the Riemann-Liouville approach. Orsingher and Beghin [23] studied the fundamental solutions to time-fractional telegraph equations of order $2 \alpha$. They obtained the Fourier transforms of the solutions for any $\alpha$ and gave a representation of their inverses in terms of stable densities. For the special case $\alpha=1 / 2$, they also showed that the fundamental solution is the probability density of a telegraph process with Brownian time. Chen et al. [9] also discussed and derived the analytical solution of the time-fractional telegraph equation with three kinds of nonhomogeneous boundary conditions, by the method of separating variables. The analytical solution of the timefractional telegraph equation considered by Chen et al. [9] is derived by Huang [13] in the whole space domain. Orsingher and Zhao [24] considered the space-fractional telegraph equations, obtaining the Fourier transform of its fundamental solution and presenting a symmetric process with discontinuous trajectories, whose transition function satisfies the space-fractional telegraph equation. Momani [22] discussed analytic and approximate solutions of the space-time fractional telegraph differential equations, with some special initial and boundary conditions, by means of the so-called Adomian decomposition method. Camargo et al. [6] discussed the so-called general space-time fractional telegraph equations by the methods of differential and integral calculus, discussing the solution by means of the Laplace and Fourier transforms in variables $t$ and $x$, respectively.

We consider the following generalized space-time fractional telegraph equation

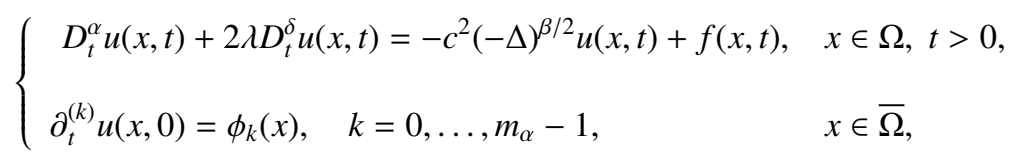

where $\Omega \subseteq \mathbb{R}^{N}, N \geq 1$, is a smooth and bounded domain; $\partial_{t}^{(k)}:=\frac{\partial^{m}}{\partial t^{m}}$, and

$$
m_{\alpha}-1<\alpha \leq m_{\alpha}, \quad m_{\delta}-1<\delta \leq m_{\delta}, \quad \alpha>\delta \geq 0, \quad m_{\alpha} \in \mathbb{N}^{*}, \quad m_{\delta} \in \mathbb{N}, \quad 0<\beta \leq 2, \quad \lambda \geq 0, \quad c \in \mathbb{R} .
$$

We also suppose that $f$ and $\phi_{k}, k=0, \ldots, m_{\alpha}-1$, are continuous functions. For $\gamma \geq 0, D^{\gamma}$ is the Caputo fractional derivative defined by

$$
D_{t}^{\gamma} u(t)= \begin{cases}\frac{1}{\Gamma(m-\gamma)} \int_{0}^{t}(t-s)^{-\gamma+m-1} u^{(m)}(s) d s, & m-1<\gamma<m, \\ u^{(m)}(t), & \gamma=m,\end{cases}
$$

where $u^{(m)}:=\frac{d^{m}}{d t^{m}} u, m \in \mathbb{N}$, and $\Gamma$ is the Euler gamma function. The fractional Laplacian operator $(-\Delta)^{\beta / 2}$ in a bounded domain is defined by (see Theorem 6 below)

$$
(-\Delta)^{\beta / 2} v(x):=\sum_{k=1}^{\infty} \lambda_{k}^{\beta / 2} \int_{\Omega} v(y) \varphi_{k}(y) d y \varphi_{k}(x), \quad v \in D\left((-\Delta)^{\beta / 2}\right),
$$

where $\left\{\lambda_{n}\right\}_{n \geq 1}$ and $\left\{\varphi_{n}\right\}_{n \geq 1}$ are the eigenvalues and eigenvectors associated to $-\Delta$ in $L^{2}(\Omega)$, and

$$
D\left((-\Delta)^{\beta / 2}\right)=\left\{v \in L^{2}(\Omega) ; \sum_{k=1}^{\infty}\left|\lambda_{k}^{\beta / 2} \int_{\Omega} v(y) \varphi_{k}(y) d y\right|^{2}<\infty\right\} .
$$

The goal of this paper is to provide analytical solutions of equation (1.1) under nonhomogeuous Dirichlet and Neumann boundary conditions. The existence is ensured via the method of separation of variables. Precisely, we prove the following theorems:

Theorem 1. (Telegraph equation with Dirichlet conditions)

Let $\Omega \subseteq \mathbb{R}^{N}$ be a smooth bounded domain. There exists an analytical solution $u$ of problem (1.1) under the following Dirichlet boundary conditions

$$
u(x, t)=g_{1}(x) g_{2}(t), \quad x \in \partial \Omega, t \geq 0,
$$

where $g_{1}$ and $g_{2}$ are nonzero smooth functions defined, respectively, on $\Omega^{c}=\mathbb{R}^{N} \backslash \Omega$ and $[0, \infty)$, and satisfying the compatibility condition $\varphi_{0}(x)=g_{1}(x) g_{2}(0)$ for all $x \in \partial \Omega$. 
Theorem 2. (Telegraph equation with Neumann conditions)

Let $\Omega \subseteq \mathbb{R}^{N}$ be a smooth, convex and bounded domain. There exists an analytical solution u of problem (1.1) under the following Neumann boundary conditions

$$
\frac{\partial u}{\partial n}(x, t)=g_{1}(x) g_{2}(t), \quad x \in \partial \Omega, t \geq 0,
$$

where $n=\vec{n}$ is the outward-pointing unit normal vector on the boundary $\partial \Omega, g_{1}$ and $g_{2}$ are nonzero smooth functions defined, respectively, on $\partial \Omega$ and $[0, \infty)$.

The organization of this paper is as follows. In Section 2, we present some definitions and basic theorems that are needed in the proofs of Theorems 1 and 2. The proofs of Theorem 1 and Theorem 2 are presented, respectively, in Section 3 and Section 4. Finally, some conclusions are given in Section 5.

\section{Basic concepts}

In this section we present the main tools concerning the fractional Laplacian and fractional derivatives that will be used throughout our analysis. We start with some definitions.

Definition 1 (see [18]). A real or complex-valued function $f(t), t>0$, is said to be in the space $C_{\alpha}, \alpha \in \mathbb{R}$, if there exists a real number $p>\alpha$ such that

$$
f(t)=t^{p} f_{1}(t)
$$

for some function $f_{1}$ in $C[0, \infty)$.

Clearly, $C_{\alpha}$ is a vector space and the set of spaces $C_{\alpha}$ is ordered by inclusion according to

$$
C_{\alpha} \subseteq C_{\beta} \Leftrightarrow \alpha \geq \beta
$$

Definition 2 (see [18]). A function $f(t), t>0$, is said to be in the space $C_{\alpha}^{m}, m \in \mathbb{N}$, if and only if $f^{(m)} \in C_{\alpha}$.

For example, the function $f$ defined by $f(t)=1, t>0$, is in $C_{0}^{1}$.

Definition 3 (see $[15,18])$. A multivariate Mittag-Leffler function $E_{\left(a_{1}, \ldots, a_{n}\right), b}\left(z_{1}, \ldots, z_{n}\right)$ of $n$ complex variables $z_{1}, \ldots, z_{n} \in$ $\mathbb{C}$ with complex parameters $a_{1}, \ldots, a_{n}, b \in \mathbb{C}$ (of positive real parts), is defined as

$$
E_{\left(a_{1}, \ldots, a_{n}\right), b}\left(z_{1}, \ldots, z_{n}\right)=\sum_{k=0}^{\infty} \sum_{\substack{l_{1}+\ldots+l_{n}=k \\ l_{1}, \ldots, l_{n} \geq 0}}\left(k ; l_{1}, \ldots, l_{n}\right) \frac{\prod_{i=1}^{n} z_{i}^{l_{i}}}{\Gamma\left(b+\sum_{i=1}^{n} a_{i} l_{i}\right)},
$$

in terms of the multinomial coefficients

$$
\left(k ; l_{1}, \ldots, l_{n}\right):=\frac{k !}{l_{1} ! \ldots l_{n} !} \quad\left(k, l_{1}, \ldots, l_{n} \in \mathbb{N}\right) .
$$

In particular, if $n=1$, the multivariate Mitteg-Leffler function is reduced to the Mitteg-Leffler function

$$
E_{a_{1}, b}\left(z_{1}\right)=\sum_{k=0}^{\infty} \frac{z_{1}^{k}}{\Gamma\left(b+k a_{1}\right)}, \quad a_{1}, b, z_{1} \in \mathbb{C} ; \mathfrak{R}\left(a_{1}\right), \mathfrak{R}(b)>0,
$$

where $\mathfrak{R}(z), z \in \mathbb{C}$, is the complex real part of $z$.

The separation of variables method used in this paper leads to two FDEs in time and space. The next theorem ensures the existence of the fractional differential equation involving the time variable. 
Theorem 3 (see [18]). Let $\mu>\mu_{1}>\ldots>\mu_{n} \geq 0, m_{i}-1<\mu_{i} \leq m_{i}, m_{i} \in \mathbb{N}, \lambda_{i} \in \mathbb{R}, i=1, \ldots, n$. Consider the initial value problem

$$
\left\{\begin{array}{l}
D_{t}^{\mu} y(t)-\sum_{i=1}^{n} \lambda_{i} D_{t}^{\mu_{i}} y(t)=g(t), \\
y^{(k)}(0)=c_{k} \in \mathbb{R}, \quad k=0, \ldots, m-1, \quad m-1<\mu \leq m,
\end{array}\right.
$$

where $g$ is assumed to lie in $C_{-1}$ if $\mu \in \mathbb{N}^{*}$ or in $C_{-1}^{1}$ if $\mu \notin \mathbb{N}^{*}$. Then (2.1) has a unique solution in the space $C_{-1}^{m}$ of the form

$$
y(t)=\int_{0}^{t} s^{\mu-1} E_{\left(\mu-\mu_{1}, \ldots, \mu-\mu_{n}\right), \mu}\left(\lambda_{1} s^{\mu-\mu_{1}}, \ldots, \lambda_{n} s^{\mu-\mu_{n}}\right) g(t-s) d s+\sum_{k=0}^{m-1} c_{k} u_{k}(t), \quad t \geq 0,
$$

with

$$
u_{k}(t)=\frac{t^{k}}{k !}+\sum_{i=l_{k}+1}^{n} \lambda_{i} t^{k+\mu-\mu_{i}} E_{\left(\mu-\mu_{1}, \ldots, \mu-\mu_{n}\right), k+1+\mu-\mu_{i}}\left(\lambda_{1} t^{\mu-\mu_{1}}, \ldots, \lambda_{n} t^{\mu-\mu_{n}}\right), \quad k=0, \ldots, m-1,
$$

fulfills the initial conditions $u_{k}^{(l)}(0)=\delta_{k l}, k, l=0, \ldots, m-1$.

Remark 1. In Theorem 3, the natural numbers $l_{k}, k=0, \ldots, m-1$, are determined from the condition $m_{l_{k}} \geq k+1$ and $m_{l_{k}+1} \leq k$. In the case $m_{i} \leq k, i=0, \ldots, m-1$, we set $l_{k}:=0$, and if $m_{i} \geq k+1, i=0, \ldots, m-1$, then $l_{k}:=n$.

The existence of the fractional differential equation involving the space variable is guaranteed through the following theorems.

Theorem 4. ([3, Theorem 3.6.1]) Let $\Omega \subseteq \mathbb{R}^{N}$ be an open bounded domain of class $C^{1}$. Then there exists a complete system $\left\{\varphi_{j}\right\}_{j=1}^{\infty}$ of eigenvectors, orthonormal in $L^{2}(\Omega)$ and orthogonal in $H_{0}^{1}(\Omega)$, and of eigenvalues $\lambda_{j}$ for the following problem

$$
\begin{cases}-\Delta u=\lambda u, & \text { in } \Omega, \\ u=0, & \text { on } \partial \Omega,\end{cases}
$$

where $D(-\Delta)=H^{2}(\Omega) \cap H_{0}^{1}(\Omega)$. Moreover, $\lambda_{j}>0, j=1, \ldots, \infty$, and $\lim _{j \rightarrow \infty} \lambda_{j}=+\infty$.

Theorem 5. ([3, Theorem 3.6.2]) Let $\Omega \subset \mathbb{R}^{N}$ be an open bounded domain of class $C^{2}$. Then there exists an orthonormal complete system $\left\{\varphi_{j}\right\}_{j=0}^{\infty}$ of eigenvectors in $L^{2}(\Omega)$ and a corresponding sequence $\lambda_{j}$ of eigenvalues for the following problem

$$
\left\{\begin{array}{l}
-\Delta u=\lambda u, \quad \text { in } \Omega \\
\frac{\partial u}{\partial n}=0, \quad \text { in } \Omega^{c}
\end{array}\right.
$$

where $D(-\Delta)=\left\{u \in H^{2}(\Omega) ; \quad \frac{\partial u}{\partial n}=0\right.$ on $\left.\partial \Omega\right\}$. Moreover, $\lambda_{j} \geq 0, j=0, \ldots, \infty$, and $\lim _{j \rightarrow \infty} \lambda_{j}=+\infty$.

The next theorem is already known in the literature (eg. [21]). However, for the sake of completeness, we hereby present the proof.

Theorem 6. Let $H$ be a Hilbert space. If $\left\{\lambda_{n}\right\}_{n \geq 1}$ and $\left\{\varphi_{n}\right\}_{n \geq 1}$ are the eigenvalues and eigenvectors associated to an operator $A$ in $H$, then $\left\{\lambda_{n}^{\alpha}\right\}_{n \geq 1}$ and $\left\{\varphi_{n}\right\}_{n \geq 1}$ are the eigenvalues and eigenvectors to the fractional operator $A^{\alpha}$, $-1<\alpha \leq 1$.

Proof. Since $H$ is a Hilbert space, we can extract an orthonormal complete system $\left\{\varphi_{i}\right\}_{i \geq 1}$ in $H$. Let $\left\{\lambda_{n}\right\}_{n \geq 1} \subseteq \mathbb{R}$ such that $\inf _{k \geq 1} \lambda_{k}>0$. For $x \in H, t \geq 0$, we define a new operator $S(t)$ as follows

$$
S(t) x=\sum_{k=1}^{\infty} e^{-\lambda_{k} t}<x, \varphi_{k}>\varphi_{k}
$$


Then $S(t)$ is a strongly continuous semigroup on $H$ whose infinitesimal generator $A$ is defined by

$$
A x=\sum_{k=1}^{\infty} \lambda_{k}<x, \varphi_{k}>\varphi_{k}
$$

and

$$
D(A)=\left\{x \in H ; \quad\|A x\|_{H}^{2}<\infty\right\}
$$

By [3, Theorem 1.4.1], we have

$$
\|A x\|_{H}^{2}=\sum_{k=1}^{\infty}\left|\lambda_{k}<x, \varphi_{k}>\right|^{2}
$$

which implies that

$$
D(A)=\left\{x \in H ; \quad \sum_{k=1}^{\infty}\left|\lambda_{k}<x, \varphi_{k}>\right|^{2}<\infty\right\} .
$$

Let $x=\varphi_{l}, l \geq 1$, then, as $\left\{\varphi_{l}\right\}_{l \geq 1}$ is an orthonormal system,

$$
A \varphi_{l}=\sum_{k=1}^{\infty} \lambda_{k}<\varphi_{l}, \varphi_{k}>\varphi_{k}=\lambda_{l} \varphi_{l}
$$

hence $\left\{\lambda_{l}\right\}_{l \geq 1}$ and $\left\{\varphi_{l}\right\}_{l \geq 1}$ are the eigenvalues and eigenvectors associated to $A$ in $H$. Let $\alpha \in(-1,1]$, we have two cases to distinguish:

- If $-1<\alpha<0$, we have (see [1])

$$
A^{\alpha} x=\frac{1}{\Gamma(-\alpha)} \int_{0}^{\infty} t^{-\alpha-1} S(t) x d t, \quad \forall x \in D\left(A^{\alpha}\right),
$$

where $D\left(A^{\alpha}\right):=\left\{x \in H ; \quad A^{\alpha-1} x \in D(A)\right\}$. Therefore

$$
\begin{aligned}
<A^{\alpha} x, \varphi_{k}> & =\frac{1}{\Gamma(-\alpha)} \int_{0}^{\infty} t^{-\alpha-1}<S(t) x, \varphi_{k}>d t \\
& =\frac{1}{\Gamma(-\alpha)} \int_{0}^{\infty} t^{-\alpha-1} e^{) \lambda_{k} t}<x, \varphi_{k}>d t \\
& =\frac{<x, \varphi_{k}>}{\Gamma(-\alpha)} \int_{0}^{\infty} t^{-\alpha-1} e^{) \lambda_{k} t} d t \\
& =\lambda_{k}^{\alpha}<x, \varphi_{k}>.
\end{aligned}
$$

This implies that

$$
A^{\alpha} x=\sum_{k=1}^{\infty} \lambda_{k}^{\alpha}<x, \varphi_{k}>\varphi_{k}
$$

Take $x=\varphi_{l}$, it follows

$$
A^{\alpha} \varphi_{l}=\sum_{k=1}^{\infty} \lambda_{k}^{\alpha}<\varphi_{l}, \varphi_{k}>\varphi_{k}=\lambda_{l}^{\alpha} \varphi_{l}
$$

- If $0<\alpha<1$,

$$
A^{\alpha} \varphi_{l}=A^{-(1-\alpha)} A \varphi_{l}=A^{-(1-\alpha)} \lambda_{l} \varphi_{l}=\lambda_{l} A^{\alpha-1} \varphi_{l}
$$

Using the first case, we get

$$
A^{\alpha-1} \varphi_{l}=\lambda_{l}^{\alpha-1} \varphi_{l}
$$

which implies

$$
A^{\alpha} \varphi_{l}=\lambda_{l}^{\alpha} \varphi_{l}
$$


The last formula still true for the cases $\alpha=0$ and $\alpha=1$. As a conclusion, $\left\{\lambda_{l}^{\alpha}\right\}_{l \geq 1}$ and $\left\{\varphi_{l}\right\}_{l \geq 1}$ are the eigenvalues and eigenvectors associated to $A^{\alpha}, \forall \alpha \in(-1,1]$. Moreover, using [3, Theorem 1.4.1], we obtain

$$
\left\|A^{\alpha} x\right\|_{H}^{2}=\sum_{k=1}^{\infty}\left|\lambda_{k}^{\alpha}<x, \varphi_{k}>\right|^{2},
$$

which yields

$$
D\left(A^{\alpha}\right)=\left\{x \in H ; \quad \sum_{k=1}^{\infty}\left|\lambda_{k}^{\alpha}<x, \varphi_{k}>\right|^{2}<\infty\right\} .
$$

\section{Proof of Theorem 1}

This section is devoted to derive the analytical solution of problem (1.1) with nonhomogeneous Dirichlet boundary conditions (1.2). Indeed, we present the

Proof of Theorem 1. We proceed in three steps.

Step 1 (Change of variables). In order to solve the problem with nonhomogeneous boundary conditions, we firstly transform it into a problem with homogeneous boundary conditions. Let

$$
u(x, t)=v(x, t)+w(x, t)
$$

where $v$ is a new unknown function and

$$
w(x, t)=y(x) g_{2}(t)
$$

with $y$ is a solution of the following $\beta$-harmonic equation

$$
\begin{cases}(-\Delta)^{\beta / 2} y(x)=0, & \text { in } \Omega, \\ y(x)=g_{1}(x), & \text { in } \Omega^{c} .\end{cases}
$$

The existence of $y$ can be found in $[12,27]$. However, in the case where $\Omega=B_{r}=B(0, r)$, the solution $y$ can be expressed explicitly by [17]

$$
y(x)=\int_{\mathbb{R}^{N} \backslash B_{r}} P(x, y) g_{1}(y) d y,
$$

where

$$
P(x, y):=C_{N, \beta}\left(\frac{r^{2}-|x|^{2}}{|y|^{2}-r^{2}}\right)^{\beta / 2} \frac{1}{|x-y|^{N}}, \quad \text { for } x \in B_{r} \text { and } y \notin B_{r},
$$

is the Poisson Kernel with $C_{N, \beta}=\Gamma(N / 2) \pi^{-N / 2-1} \sin (\pi \beta / 2)$.

The function $w$ satisfies the boundary conditions

$$
w(x, t)=y(x) g_{2}(t)=g_{1}(x) g_{2}(t)=u(x, t), \text { for all } x \in \partial \Omega, t \geq 0,
$$

which implies

$$
v(x, t)=u(x, t)-w(x, t)=0, \text { for all } x \in \partial \Omega, t \geq 0 .
$$

Therefore $v(x, t)$ satisfies the following problem with homogeneous boundary conditions

$$
\begin{cases}D_{t}^{\alpha} v(x, t)+2 \lambda D_{t}^{\delta} v(x, t)=-c^{2}(-\Delta)^{\beta / 2} v(x, t)+\tilde{f}(t, x), & x \in \Omega, t>0, \\ \partial_{t}^{(k)} v(x, 0)=\tilde{\phi}_{k}(x), \quad k=0, \ldots, m_{\alpha}-1, & x \in \bar{\Omega}, \\ v(x, t)=0, & x \in \partial \Omega, t \geq 0,\end{cases}
$$


where

$$
\begin{aligned}
\tilde{f}(t, x) & =-D_{t}^{\alpha} w(x, t)-2 \lambda D_{t}^{\delta} w(x, t)+f(t, x), \\
\tilde{\varphi}_{k}(x) & =\varphi_{k}(x)-\partial_{t}^{(k)} w(x, 0), \quad k=0, \ldots, m_{\alpha}-1 .
\end{aligned}
$$

Step 2 (Separation of variables). We solve problem, (3.3) with zero function replacing $\tilde{f}(t, x)$, by the method of separation of variables. If we let $v(t, x)=X(x) T(t)$ and substitute it in (3.3), we obtain a fractional differential equation in $X(x)$ :

$$
\begin{cases}(-\Delta)^{\beta / 2} X(x)=\frac{a}{c^{2}} X(x), & \text { in } \Omega, \\ X(x)=0, & \text { on } \partial \Omega,\end{cases}
$$

and a fractional linear differential equation with the Caputo derivative in $T(t)$ :

$$
D_{t}^{\alpha} T(t)+2 \lambda D_{t}^{\delta} T(t)+a T(t)=0,
$$

where the parameter $a$ is a positive constant. Applying Theorem 6 with $A=-\Delta, \alpha=\beta / 2, H=L^{2}(\Omega)$ and $D(A)=$ $H^{2}(\Omega) \cap H_{0}^{1}(\Omega)$, there exists $\left\{\lambda_{n}^{\beta / 2}\right\}_{n \geq 1},\left\{\varphi_{n}\right\}_{n \geq 1}$ eigenvalues and eigenvectors of problem (3.4) where $\left\{\lambda_{n}\right\}_{n \geq 1},\left\{\varphi_{n}\right\}_{n \geq 1}$ are the eigenvalues and eigenvectors of (3.4) with $\beta=2$ (see Theorem 4). Note that for $a=a_{n}, n \geq 1$, we have $X=X_{n}=\varphi_{n}$ and $\frac{a_{n}}{c^{2}}=\lambda_{n}^{\beta / 2}$.

We now seek a solution of (3.3) of the form

$$
v(t, x)=\sum_{n=1}^{\infty} B_{n}(t) \varphi_{n}(x),
$$

where we assume that the series can be differentiated term by term. In order to determine $B_{n}(t)$, we expand $\tilde{f}(t, x)$ in the orthonormal complete system $\left\{\varphi_{n}\right\}_{n \geq 1}$ (see proof of Theorem 6):

$$
\tilde{f}(t, x)=\sum_{n=1}^{\infty} \tilde{f}_{n}(t) \varphi_{n}(x),
$$

where

$$
\tilde{f}_{n}(t)=<\tilde{f}(t, x), \varphi_{n}(x)>=\int_{\Omega} \tilde{f}(t, x) \varphi_{n}(x) d x .
$$

Substituting (3.6), (3.7) into (3.3) yields

$$
\sum_{n=1}^{\infty} \varphi_{n}(x)\left[D_{t}^{\alpha} B_{n}(t)+2 \lambda D_{t}^{\delta} B_{n}(t)\right]=\sum_{n=1}^{\infty} \varphi_{n}(x)\left[-a_{n} B_{n}(t)+\tilde{f}_{n}(t)\right],
$$

where we have used the fact that $\varphi_{n}(x)$ is a solution of (3.4). Since $\left\{\varphi_{n}\right\}_{n \geq 1}$ is an orthonormal system, multiplying both members of (3.8) by $\varphi_{n}$ and integrating on $\mathbb{R}^{N}$ lead to

$$
D_{t}^{\alpha} B_{n}(t)+2 \lambda D_{t}^{\delta} B_{n}(t)+a_{n} B_{n}(t)=\tilde{f}_{n}(t) .
$$

On the other hand, since $v(t, x)$ satisfies the initial conditions in (3.3), we must have

$$
\sum_{n=1}^{\infty} \partial_{t}^{(k)} B_{n}(0) \varphi_{n}(x)=\tilde{\varphi}_{k}(x), \quad k=0, \ldots, m_{\alpha}-1 \quad x \in \bar{\Omega},
$$

which implies

$$
\partial_{t}^{(k)} B_{n}(0)=<\tilde{\varphi}_{k}(x), \varphi_{n}(x)>=\int_{\Omega} \tilde{\varphi}_{k}(x) \varphi_{n}(x) d x=: c_{k, n}, \quad n \geq 1, k=0, \ldots, m_{\alpha}-1 .
$$

Finally, for each value of $n,(3.9)$ and (3.10) constitute a fractional initial value problem. 
Step 3 (Analytical solution). According to Theorem 3, the fractional initial value problem (3.9)-(3.10) has the analytical solution

$$
B_{n}(t)=\int_{0}^{t} s^{\alpha-1} E_{(\alpha-\delta, \alpha), \alpha}\left(-2 \lambda s^{\alpha-\delta},-a_{n} s^{\alpha}\right) \tilde{f}_{n}(t-s) d s+\sum_{k=0}^{m_{\alpha}-1} c_{k, n} v_{k}(t), \quad t \geq 0
$$

where

$$
v_{k}(t)=\frac{t^{k}}{k !}+\sum_{i=l_{k}+1}^{2} \xi_{i} t^{k+\alpha-\mu_{i}} E_{(\alpha-\delta, \alpha), k+1+\alpha-\mu_{i}}\left(\xi_{1} t^{\alpha-\delta}, \xi_{2} t^{\alpha}\right), \quad k=0, \ldots, m_{\alpha}-1,
$$

with $\xi_{1}:=-2 \lambda, \xi_{2}=-a_{n}, \mu_{1}=\delta$ and $\mu_{2}=0$. Note that $l_{k}, k=0, \ldots, m_{\alpha}-1$, are as in Remark 1 . Hence we get the analytical solution of the initial boundary value problem (3.3) under the form

$$
v(t, x)=\sum_{n=1}^{\infty} B_{n}(t) \varphi_{n}(x)=\sum_{n=1}^{\infty}\left[\int_{0}^{t} s^{\alpha-1} E_{(\alpha-\delta, \alpha), \alpha}\left(-2 \lambda s^{\alpha-\delta},-a_{n} s^{\alpha}\right) \tilde{f}_{n}(t-s) d s+\sum_{k=0}^{m_{\alpha}-1} c_{k, n} v_{k}(t)\right] \varphi_{n}(x),
$$

where $v_{k}(t)$ are given in (3.12), $k=0, \ldots, m_{\alpha}-1$. Therefore the analytical solution of (1.1)-(1.2) is:

$$
u(t, x)=v(t, x)+w(t, x)
$$

where $v(t, x)$ and $w(t, x)$ are, respectively, given by (3.13) and (3.1).

Remark 2. In problem (3.2), we had to work with boundary conditions defined on $\Omega^{c}$ and not only on $\partial \Omega$ since the following $\beta$-harmonic equation

$$
\begin{cases}(-\Delta)^{\beta / 2} y(x)=0, & \text { in } \Omega \\ y(x)=g_{1}(x), & \text { in } \partial \Omega .\end{cases}
$$

is ill-posed (see $[5,17,26]$. In fact, the probabilistic solution gives the best insight: $(-\Delta)^{\beta / 2}$ generates a jump process

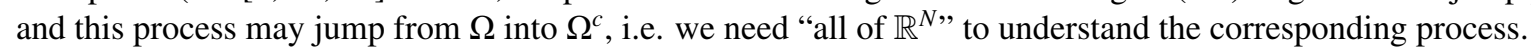

Remark 3. In Theorem 1, the condition on the function $g_{1}$ being defined over $\Omega^{c}$ and not on $\partial \Omega$ may seem strange at the first glance. However, this condition was set in order to be compatible with that required (see the remark above) for the existence of (3.2). In fact, assuming some regularity on the set $\Omega$ allows us to define $g_{1}$ on $\partial \Omega$ and to extend it suitably over $\Omega^{c}$. For example, if $\Omega$ is smooth, convex and bounded, we may choose the same extension as in Step 1 of the proof of Theorem 2 (see Section 4 below)

\subsection{Special Cases}

The solution of (1.1)-(1.2) can be considered as a generalized solution for a wide variety of evolution equations. However, we only list here two special cases:

Case 1. Take $\alpha=2 \delta, m_{\alpha}=2, m_{\delta}=1, \beta=2, N=1, \Omega=(0, L)$. Then the eigenvalues and the eigenvectors can be expressed as follows:

for all $n \geq 1$. Therefore

$$
a_{n}=\left(\frac{n \pi}{L}\right)^{2} \quad \text { and } \quad \varphi_{n}(x)=\sqrt{\frac{2}{L}} \sin \left(\frac{n \pi}{L} x\right)
$$

$$
v_{0}(t)=1-\left(\frac{n \pi}{L}\right)^{2} t^{2 \delta} E_{(\delta, 2 \delta), 1+2 \delta}\left(-2 \lambda t^{\delta},-\left(\frac{n \pi}{L}\right)^{2} t^{2 \delta}\right)
$$

and

$$
v_{1}(t)=t-2 \lambda t^{1+\delta} E_{(\delta, 2 \delta), 2+\delta}\left(-2 \lambda t^{\delta},-\left(\frac{n \pi}{L}\right)^{2} t^{2 \delta}\right)-\left(\frac{n \pi}{L}\right)^{2} t^{1+2 \delta} E_{(\delta, 2 \delta), 2+2 \delta}\left(-2 \lambda t^{\delta},-\left(\frac{n \pi}{L}\right)^{2} t^{2 \delta}\right) .
$$


Moreover the particular solution $w$ of (3.1) can be given by

$$
w(t, x)= \begin{cases}g_{1}(0) g_{2}(t)+\frac{\left(g_{1}(L) g_{2}(t)-g_{1}(0) g_{2}(t)\right) x}{L}, & \text { for } x \in[0, L], \\ g_{1}(L) g_{2}(t), & \text { for } x \geq L, \\ g_{1}(0) g_{2}(t), & \text { for } x \leq 0 .\end{cases}
$$

The analytical solution of (1.1)-(1.2) is

$$
u(t, x)=v(t, x)+w(t, x)
$$

where

$$
v(t, x)=\sqrt{\frac{2}{L}} \sum_{n=1}^{\infty}\left[\int_{0}^{t} s^{2 \delta-1} E_{(\delta, 2 \delta), 2 \delta}\left(-2 \lambda s^{\delta},-\left(\frac{n \pi}{L}\right)^{2} s^{2 \delta}\right) \tilde{f}_{n}(t-s) d s+B_{n}(0) v_{0}(t)+B_{n}^{\prime}(0) v_{1}(t)\right] \sin \left(\frac{n \pi}{L} x\right),
$$

with

$$
B_{n}(0)=\sqrt{\frac{2}{L}} \int_{0}^{L} \tilde{\varphi}_{0}(x) \sin \left(\frac{n \pi}{L} x\right) d x, \quad B_{n}^{\prime}(0)=\sqrt{\frac{2}{L}} \int_{0}^{L} \tilde{\varphi}_{1}(x) \sin \left(\frac{n \pi}{L} x\right) d x
$$

and

$$
\tilde{f}_{n}(t)=\sqrt{\frac{2}{L}} \int_{0}^{L} \tilde{f}(t, x) \sin \left(\frac{n \pi}{L} x\right) d x .
$$

This result is in accord with the result obtained in [9, Section 3].

Case 2. Take $\alpha=2, \delta=1, m_{\alpha}=2, m_{\delta}=1, \beta=2, \lambda=0, N=1, \Omega=(0, L)$. As above, one can choose the following expression as the eigenvalues and the eigenvectors respectively:

$$
a_{n}=\left(\frac{n \pi}{L}\right)^{2} \quad \text { and } \quad \varphi_{n}(x)=\sqrt{\frac{2}{L}} \sin \left(\frac{n \pi}{L} x\right),
$$

for all $n \geq 1$. Therefore

$$
v_{0}(t)=1-\left(\frac{n \pi}{L}\right)^{2} t^{2} E_{(1,2), 3}\left(0,-\left(\frac{n \pi}{L}\right)^{2} t^{2}\right)=1-\left(\frac{n \pi}{L}\right)^{2} t^{2} E_{2,3}\left(-\left(\frac{n \pi}{L}\right)^{2} t^{2}\right)=\cos \left(\frac{n \pi}{L} t\right)
$$

and

$$
v_{1}(t)=t-\left(\frac{n \pi}{L}\right)^{2} t^{3} E_{(1,2), 4}\left(0,-\left(\frac{n \pi}{L}\right)^{2} t^{2}\right)=t-\left(\frac{n \pi}{L}\right)^{2} t^{3} E_{2,4}\left(-\left(\frac{n \pi}{L}\right)^{2} t^{2}\right)=\frac{L}{n \pi} \sin \left(\frac{n \pi}{L} t\right)
$$

Since

the solution is given by

$$
s E_{(1,2), 2}\left(0,-\left(\frac{n \pi}{L}\right)^{2} s^{2}\right)=\sin \left(\frac{n \pi}{L} s\right)
$$

$$
u(t, x)=v(t, x)+w(t, x)
$$

with

$$
v(t, x)=\sqrt{\frac{2}{L}} \sum_{n=1}^{\infty}\left[\int_{0}^{t} \sin \left(\frac{n \pi}{L} s\right) \tilde{f}_{n}(t-s) d s+B_{n}(0) v_{0}(t)+B_{n}^{\prime}(0) v_{1}(t)\right] \sin \left(\frac{n \pi}{L} x\right),
$$

where $B_{n}(0), B_{n}^{\prime}(0)$ and $\tilde{f}_{n}(t)$ are, respectively, given by (3.14), (3.15) and (3.16). Indeed, this result is the solution of the wave equation. 


\section{Proof of Theorem 2}

In this section, we derive the analytical solution of problem (1.1) with nonhomogeneous Neumann boundary conditions (1.3).

Proof of Theorem 2. We proceed in four steps.

Step 1 ( $\beta$-harmonic equation with Neumann conditions). Consider the particular extension $\xi$ of $g_{1}$ over $\Omega^{c}$ :

$$
\xi(x)=g_{1}(\bar{x})|x-\bar{x}|, \quad x \in \Omega^{c}
$$

where $\bar{x}:=\operatorname{Pr}_{\bar{\Omega}}(x)$ is the orthogonal projection of $x$ on $\bar{\Omega}$. This function is well-defined by the uniqueness of $\bar{x}$ due to the convexity of $\Omega$. Then, for all $z \in \partial \Omega$, we have

$$
\begin{aligned}
\frac{\partial \xi}{\partial n}(z) & =\lim _{h \rightarrow 0} \frac{\xi(z+h \vec{n})-\xi(z)}{h} \\
& =\lim _{h \rightarrow 0} \frac{g_{1}(\overline{z+h \vec{n}})|(z+h \vec{n})-(\overline{z+h \vec{n}})|-g_{1}(\bar{z})|z-\bar{z}|}{h} \\
& =\lim _{h \rightarrow 0} \frac{h g_{1}(z)-0}{h} \\
& =g_{1}(z),
\end{aligned}
$$

where we have used the fact that $\overline{z+h \vec{n}}=\bar{z}=z$. Let $y$ be a solution of the following $\beta$-harmonic equation with Dirichlet conditions

$$
\begin{cases}(-\Delta)^{\beta / 2} y(x)=0, & \text { in } \Omega \\ y(x)=\xi(x), & \text { in } \Omega^{c}\end{cases}
$$

Note that

$$
\frac{\partial y}{\partial n}(z)=\frac{\partial \xi}{\partial n}(z)=g_{1}(z), \quad z \in \partial \Omega
$$

This yields that $y$ is a solution of the following $\beta$-harmonic equation with Neumann conditions

$$
\begin{cases}(-\Delta)^{\beta / 2} y=0, & \text { in } \Omega, \\ \frac{\partial y}{\partial n}=g_{1}, & \text { on } \partial \Omega .\end{cases}
$$

Step 2 (Change of variables). We argue in a similar manner as in Step 1 of the proof of Theorem 1.1. In fact, let

$$
u(x, t)=v(x, t)+w(x, t)
$$

where

$$
w(x, t)=y(x) g_{2}(t)
$$

with $y$ is a solution of (4.1) and consequently

$$
\frac{\partial w}{\partial n}(x, t)=g_{1}(x) g_{2}(t), \quad \text { for all } x \in \partial \Omega, t \geq 0 .
$$

The function $v(x, t)$ is an unknown function satisfying the following problem with homogeneous boundary conditions

$$
\begin{cases}D_{t}^{\alpha} v(x, t)+2 \lambda D_{t}^{\delta} v(x, t)=-c^{2}(-\Delta)^{\beta / 2} v(x, t)+\tilde{f}(t, x), & x \in \Omega, t>0, \\ \partial_{t}^{(k)} v(x, 0)=\tilde{\phi}_{k}(x), \quad k=0, \ldots, m_{\alpha}-1, & x \in \bar{\Omega}, \\ \frac{\partial v}{\partial n}(x, t)=0, & x \in \partial \Omega, t \geq 0,\end{cases}
$$


where

$$
\begin{aligned}
\tilde{f}(t, x) & =-D_{t}^{\alpha} w(x, t)-2 \lambda D_{t}^{\delta} w(x, t)+f(t, x), \\
\tilde{\varphi}_{k}(x) & =\varphi_{k}(x)-\partial_{t}^{(k)} w(x, 0), \quad k=0, \ldots, m_{\alpha}-1 .
\end{aligned}
$$

Step 3 (Separation of variables). Let $v(t, x)=X(x) T(t)$ (supposing that $\tilde{f}(t, x)=0$ ) and substitute it in (4.3), we get the following fractional differential equation with Neumann boundary conditions:

$$
\begin{cases}(-\Delta)^{\beta / 2} X=\frac{a}{c^{2}} X, & \text { in } \Omega, \\ \frac{\partial X}{\partial n}=0, & \text { on } \partial \Omega .\end{cases}
$$

Applying Theorem 6 with $A=-\Delta, \alpha=\beta / 2, H=L^{2}(\Omega)$ and $D(A)=\left\{u \in H^{2}(\Omega) ; \quad \frac{\partial u}{\partial n}=0\right.$ on $\left.\partial \Omega\right\}$, there exists $\left\{\lambda_{n}^{\beta / 2}\right\}_{n \geq 1},\left\{\varphi_{n}\right\}_{n \geq 1}$ eigenvalues and eigenvectors of (4.4) where $\left\{\lambda_{n}\right\}_{n \geq 1},\left\{\varphi_{n}\right\}_{n \geq 1}$ are the eigenvalues and eigenvectors of (4.4) with $\beta=2$ (see Theorem 5). Note that for $a=a_{n}, n \geq 1$, we have $X=X_{n}=\varphi_{n}$ and $\frac{a_{n}}{c^{2}}=\lambda_{n}^{\beta / 2}$. Hence, the solution of (4.3) has the form

$$
v(t, x)=\sum_{n=1}^{\infty} B_{n}(t) \varphi_{n}(x) .
$$

By repeating the same argument as in Step 2 of the proof of Theorem 1.1, we conclude that

$$
\left\{\begin{array}{l}
D_{t}^{\alpha} B_{n}(t)+2 \lambda D_{t}^{\delta} B_{n}(t)+a_{n} B_{n}(t)=\tilde{f}_{n}(t) \\
\partial_{t}^{(k)} B_{n}(0)=\int_{\Omega} \tilde{\varphi}_{k}(x) \varphi_{n}(x) d x=: c_{k, n}, \quad n \geq 1, k=0, \ldots, m_{\alpha}-1 .
\end{array}\right.
$$

Step 4 (Analytical solution). The analytical solution of (1.1)-(1.3) is

$$
u(t, x)=v(t, x)+w(t, x),
$$

where $v(t, x)$ and $w(t, x)$ are given by (3.13) and (4.2) respectively.

\subsection{A Special Case}

We give a special case of the solution of (1.1)-(1.3). Take $\alpha=2 \delta, m_{\alpha}=2, m_{\delta}=1, \beta=2, N=1, \Omega=(0, L)$ and $g_{1}=C$ is a constant function. Then the eigenvalues and the eigenvectors of problem (4.4) can be expressed as follows:

for all $n \geq 1$. Therefore

$$
a_{n}=\left(\frac{n \pi}{L}\right)^{2} \quad \text { and } \quad \varphi_{n}(x)=\sqrt{\frac{2}{L}} \cos \left(\frac{n \pi}{L} x\right),
$$

$$
v_{0}(t)=1-\left(\frac{n \pi}{L}\right)^{2} t^{2 \delta} E_{(\delta, 2 \delta), 1+2 \delta}\left(-2 \lambda t^{\delta},-\left(\frac{n \pi}{L}\right)^{2} t^{2 \delta}\right)
$$

and

$$
v_{1}(t)=t-2 \lambda t^{1+\delta} E_{(\delta, 2 \delta), 2+\delta}\left(-2 \lambda t^{\delta},-\left(\frac{n \pi}{L}\right)^{2} t^{2 \delta}\right)-\left(\frac{n \pi}{L}\right)^{2} t^{1+2 \delta} E_{(\delta, 2 \delta), 2+2 \delta}\left(-2 \lambda t^{\delta},-\left(\frac{n \pi}{L}\right)^{2} t^{2 \delta}\right) .
$$

Moreover the particular solution $w$ of (3.1) is given by

$$
w(t, x)=C x g_{2}(t) \quad x \in[0, L], t \geq 0 .
$$

The analytical solution is

$$
u(t, x)=v(t, x)+w(t, x)
$$

where

$$
v(t, x)=\sqrt{\frac{2}{L}} \sum_{n=1}^{\infty}\left[\int_{0}^{t} s^{2 \delta-1} E_{(\delta, 2 \delta), 2 \delta}\left(-2 \lambda s^{\delta},-\left(\frac{n \pi}{L}\right)^{2} s^{2 \delta}\right) \tilde{f}_{n}(t-s) d s+B_{n}(0) v_{0}(t)+B_{n}^{\prime}(0) v_{1}(t)\right] \cos \left(\frac{n \pi}{L} x\right),
$$


with

$$
B_{n}(0)=\sqrt{\frac{2}{L}} \int_{0}^{L} \tilde{\varphi}_{0}(x) \cos \left(\frac{n \pi}{L} x\right) d x, \quad B_{n}^{\prime}(0)=\sqrt{\frac{2}{L}} \int_{0}^{L} \tilde{\varphi}_{1}(x) \cos \left(\frac{n \pi}{L} x\right) d x
$$

and

$$
\tilde{f}_{n}(t)=\sqrt{\frac{2}{L}} \int_{0}^{L} \tilde{f}(t, x) \cos \left(\frac{n \pi}{L} x\right) d x .
$$

This result is in accord with the result obtained in [9, Section 4].

\section{Conclusions}

In this paper we have considered the nonhomogeneous space-time fractional telegraph equation (1.1) under Dirichlet and Neumann boundary conditions (1.2) and (1.3), respectively. We have derived the analytical solutions of problems (1.1)-(1.2) and (1.1)-(1.3) using the separation of variables method. The time fractional derivative is considered in the Caputo sense and the space fractional derivative in the sense of fractional Laplacian. The general solutions, which are given in the form of the multivariate Mittag-Leffler function, reduce to those of the classical telegraph equation and corresponding diffusion and wave equations.

\section{Acknowledgments}

The authors are greatly indebted to the Lebanese Association for Scientific Research (LASeR) and to the School of Arts and Sciences at the Lebanese International University (LIU) for their kind hospitality during the achievement of this work. They would like to express their sincere thanks to Professor Mokhtar Kirane for proposing the problem. The first author was supported by a grant of the LASeR.

\section{References}

[1] W. Arendt, Ch. Batty, M. Hieber Vector-Valued Laplace Transforms and Cauchy Problems, Monographs in Mathematics Vol. 96, Birkhaeuser Verlag, 2001

[2] R. L. Bagley, On the fractional order initial value problem and its engineering applications, in: Fractional Calculus and Its Applications (Ed. K. Nishimoto), Tokyo, College of Engineering, Nihon University, 1990, 12-20.

[3] V. Barbu, Partial differential equations and boundary value problems, 1998, $292 \mathrm{pp}$.

[4] H. Beyer, S. Kempfle, Definition of physically consistent damping laws with fractional derivatives, ZAMM 75 (1995), $623-635$.

[5] J. Bliedtner, W. Hansen, Potential Theory - An Analytic and Probabilistic Approach to Balayage, Universitext, Springer Verlag, Berlin 1986.

[6] R. Figueiredo Camargo, A. O. Chiacchio, E. Capelas de Oliveira, Differentiation to fractional orders and the fractional telegraph equation, Journal of Mathematical Physics, vol. 49, no. 3, Article ID 033505, 12 pages, 2008.

[7] M. Caputo, F. Mainardi, Linear models of dissipation in anelastic solids, Riv. Nuovo Cimento (Ser II) 1 (1971), 161-198.

[8] R. C. Cascaval, E. C. Eckstein, C. L. Frota, J. A. Goldstein, Fractional telegraph equations, Journal of Mathematical Analysis and Applications, 276 (2002), 145-159.

[9] J. Chen, F. Liu, V. Anh, Analytical solution for the time-fractional telegraph equation by the method of separating variables, J. Math. Anal. Appl. 338 (2008), 1364-1377.

[10] R. Gorenflo, F. Mainardi, Fractional calculus: integral and differential equations of fractional order, in: Fractals and Fractional Calculus in Continuum Mechanics (Eds. A. Carpinteri and F. Mainardi), Springer Verlag, Wien and New York, 1997, $223-276$.

[11] R. Gorenflo, R. Rutman, On ultraslow and intermediate processes, in: Transform Methods and Special Functions (Eds. P. Rusev, I. Dimovski, V. Kiryakova), Science Culture Technology Publishing (SCTP), Singapore, 1995, 61-81.

[12] Q.-Y. Guan, Z.-M. Ma, Boundary Problems for Fractional Laplacians, Stoch. Dyn. 5 (2005), $385-424$

[13] F. Huang, Analytical solution for the time-fractional telegraph equation, J. Appl. Math., vol. 2009, Article ID 890158, 9 pages, 2009.

[14] N. Jacob, V. Knopova, Fractional derivatives and fractional powers as tools in understanding Wentsell boundary value problems for pseudodifferential operators generating Markov processes, Frac. Calculus and Applied Analysis 8 (2005),

[15] A. A. Kilbas, H. M. Srivastava, J. J. Trujillo, Theory and Applications of Fractional Differential Equations, Volume 204 (North-Holland Mathematics Studies), 2006.

[16] J. Lamperti, Probability, Benjamin, New York, 1966.

[17] N. S. Landkof, Foundations of Modern Potential Theory, Grundlehren der mathematischen Wissenschaften, Vol. 180, Springer Verlag, Berlin 1972.

[18] Y. Luchko, R. Gorenflo, An operational method for solving fractional differential equations with the Caputo derivatives, Acta Math. Vietnam. 24 (1999), 207-233. 
[19] F. Mainardi, Fractional relaxation and fractional diffusion equations, mathematical aspects, in: Proceedings of the 12-th IMACS World Congress (Ed. W.F. Ames), Georgia Tech Atlanta, Vol. 1, (1994), 329-332.

[20] F. Mainardi, Fractional calculus: some basic problems in continuum and statistical mechanics, in: Fractals and Fractional Calculus in Continuum Mechanics (Eds. A. Carpinteri and F. Mainardi), Springer Verlag, Wien and New York, 1997, 291-348.

[21] Robert C. Mcowen, Partial differential equation, second edition, Methods and Applications, 2003.

[22] S. Momani, Analytic and approximate solutions of the space- and time-fractional telegraph equations, Applied Mathematics and Computation, 170 (2005), 1126-1134.

[23] E. Orsingher, L. Beghin, Time-fractional telegraph equation and telegraph processes with Brownian time, Probab. Theory Related Fields 128 (2004) 141-160.

[24] E. Orsingher, X. Zhao, The space-fractional telegraph equation and the related fractional telegraph process, Chinese Annals of Mathematics Series B, 24 (2003), 45-56.

[25] I. Podlubny, Fractional Differential Equations: An Introduction to Fractional Derivatives, Fractional Differential Equations, to Methods of Their Solution and Some of Their Applications, vol. 198 of Mathematics in Science and Engineering, Academic Press, San Diego, Calif, USA, 1990

[26] M. Riesz, Intégrales de Riemann-Liouville et potentiels, Acta Sci. Szeged 9 (1938), 1-42.

[27] L. E. Silvestre, Regularity of the obstacle problem for a fractional power of the Laplace operator, Ph.D. University of Texas at Austin, May 2005. 